\title{
Natural Convection and Entropy Generation in Partially Heated Porous Wavy Cavity Saturated by a Nanofluid
}

\author{
Ali J. Chamkha ${ }^{1}$, Ammar I. Alsabery ${ }^{2,3}$, Nader Nader ${ }^{1}$, Ali Al-Mudhaf ${ }^{4}$ \\ ${ }^{1}$ Mechanical Engineering Department, Prince Mohammad Bin Fahd University \\ Al-Khobar 31952, Saudi Arabia \\ achamkha@pmu.edu.sa \\ ${ }^{2}$ Refrigeration \& Air-conditioning Technical Engineering Department, the Islamic University \\ Najaf, Iraq \\ ${ }^{3}$ School of Mathematical Sciences, Universiti Kebangsaan Malaysia \\ 43600 UKM Bangi, Selangor, Malaysia \\ ${ }^{4}$ Manufacturing Engineering Department, Public Authority for Applied Education and Training \\ Shuweikh 70654, Kuwait
}

\begin{abstract}
Steady laminar natural convection and entropy generation in a porous wavy cavity saturated by a nanofluid with partial bottom temperature is studied numerically using the finite element method. An isothermal heater is placed on the bottom horizontal wall of the cavity with length $h$ while the wavy vertical walls are maintained at the same constant cold temperature $T_{c}$. The remainder of the bottom wall and the top wall are kept adiabatic. The boundaries of the domain are assumed to be impermeable and the fluid within the cavity is a water-based nanofluid having $\mathrm{Cu}$ nanoparticles. The Boussinesq approximation is applicable and the Tiwari and Das' nanofluid model is considered. The Forchheimer-Brinkman-extended Darcy model is assumed to hold. The numerical computations are obtained for various values of Darcy number, nanoparticle volume fraction and the amplitude of the wave. Based on the obtained results, it is found that the strength of the streamlines increases with the increment of the Darcy number.
\end{abstract}

Keywords: Natural Convection, Entropy Generation, Wavy Porous Cavity, Non-Darcy Model, Partial Heating.

\section{Introduction}

Convection heat transfer in porous medium a significant phenomenon in science and engineering systems due to wide range of industrial applications such as geothermal reservoirs, waste nuclear processing, matrix heat exchangers, optimization of solidification processes of metals and alloys, float glass production, flow and heat transfer in solar ponds, air conditioning in rooms, optimization of solidification processes of metals and alloys, dissemination control of chemical waste and pollutants, electronic packages, grain storage systems and many others [1]. Natural convection and heat transfer in closed cavities with different shapes takes a large part of the heat transfer literature. Square, rectangle, triangle, cylindrical, elliptical and spherical geometries have been studied by many researchers. Complex geometries cover different types of geometrical configurations, namely wavy walls cavities, concave and convex curved walls cavities, etc. A very recent comprehensive literature survey concerning convection heat transfer in wavy porous cavities is given by Shenoy et al. [2]. The authors of this book gave an excellent background in the field of convective heat transfer in wavy cavities filled with viscous fluids, porous media, and nanofluids. Alsabery et al. [3] numerically studied the Effects of nonuniform heating and wall conduction on natural convection heat transfer in a square porous cavity using local thermal nonequilibrium (LTNE) model.

The study of convective heat transfer problems within complex geometries have received a considerable attention in the literature due to its wide applications in engineering problems such as; solar collectors, micro-electronic devices, electrical and nuclear components, etc. The problem of natural convection heat transfer in a wavy cavity was considered by Das and Mahmud [4]. They found that the number of undulations of the wavy wall is clearly affected the heat transfer characteristics within the cavity. Rostami [5] numerically studied the unsteady fluid flow and heat transfer problem in a cavity with vertical wavy walls and horizontal straight walls. Natural convection in wavy porous cavities under the influence of thermal radiation and with the use of thermal non-equilibrium model is studied by Mansour et al. [6]. 
A nanofluid is defined as a smart fluid with suspended nanoparticles of average sizes below $100 \mathrm{~nm}$ in conventional heat transfer fluids such as water, oil, and ethylene glycol. A nanofluid as a working medium has been considered by many researchers for the simple reason that it has the presence of nanoparticles resulting in higher thermal conductivity of medium and the heat transfer becoming enhanced. There has been a surge in research activities concerning natural convective heat transfer in nanofluid-saturated porous media. The work of Sun and Pop [7] considered the natural convection heat transfer in a triangular cavity heated by a wall heater and filled with a porous medium and saturated with different nanofluids types. Chamkha and Ismael [8] studied the conjugate natural convective heat transfer in a porous cavity filled with nanofluids and heated by a triangular thick wall. Abu-Nada and Chamkha [9] numerically studied the effects of lid-driven wall on mixed convection flow in a lid-driven cavity with a wavy wall filled with a nanofluid. They found that heat transfer rate increases with the increment of the volume fraction of nanoparticles for all values of Richardson numbers. Recently, the problem of natural convection in a partially heated wavy porous cavity filled with a nanofluid under the effects of Brownian diffusion and thermophoresis was investigated by Sheremet et al. [10]. They found that the local heat source has an efficient influence of the nanofluid flow and heat transfer rate. Nevertheless, the study of natural convection and entropy generation in partially heated porous wavy cavity saturated by a nanofluid. Therefore, the aim of this study is to investigate the natural convection heat transfer and entropy generation in a porous wavy cavity saturated by a nanofluid with partial bottom temperature.

\section{Mathematical Formulation}

The steady two-dimensional natural convection problem in a wavy cavity with a length $L$, is illustrated in Fig. 1 . An isothermal heater is placed on the bottom horizontal wall of the cavity with length $h$ while the wavy vertical walls are maintained at the same constant cold temperature $T_{c}$. The remainder of the bottom wall and the top wall are kept adiabatic. The boundaries of the domain are assumed to be impermeable, the fluid within the cavity is a water-based nanofluid having $\mathrm{Cu}$ nanoparticles. The Boussinesq approximation is applicable and the Tiwari and Das' with Maxwell-Garnett and Brinkman models are used for modelling the nanofluid heat transfer. The Forchheimer-Brinkman-extended Darcy model is assumed to hold. The governing equations for natural convection flow using conservation of mass, momentum and energy equations can be written in dimensionless form as follows:

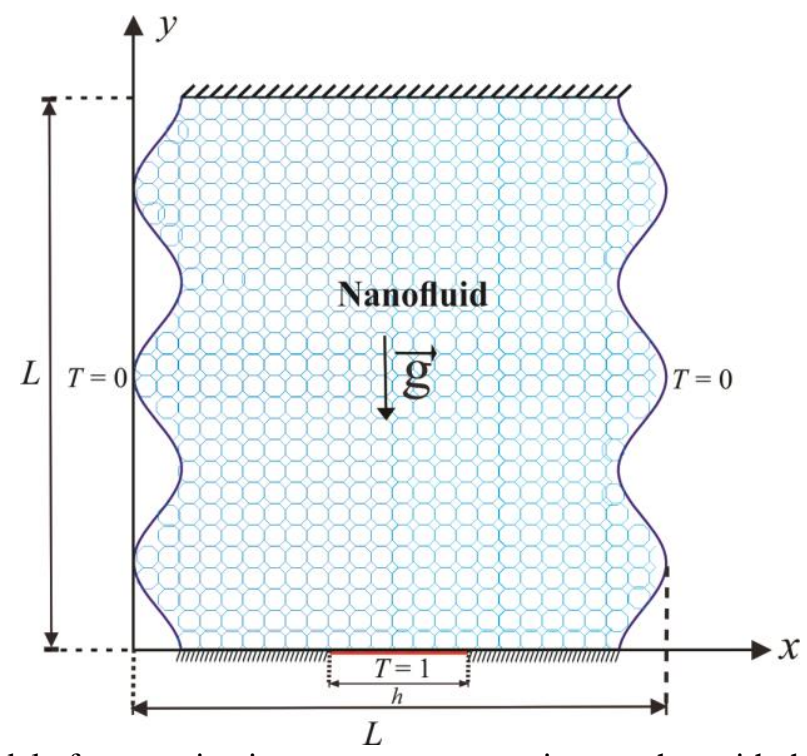

Fig. 1: Physical model of convection in a wavy porous cavity together with the coordinate system.

$$
\frac{\partial U}{\partial X}+\frac{\partial V}{\partial Y}=0
$$




$$
\begin{aligned}
U \frac{\partial U}{\partial X}+V \frac{\partial U}{\partial Y}= & -\frac{C_{F}}{\sqrt{D a} \varepsilon^{3 / 2}} U \sqrt{U^{2}+V^{2}}+\frac{\operatorname{Pr}_{f}\left(\mu_{n f} / \mu_{f}\right)}{\phi\left(\rho_{p} / \rho_{f}\right)+(1-\phi)}\left(\frac{\partial^{2} U}{\partial X^{2}}+\frac{\partial^{2} U}{\partial Y^{2}}\right) \\
& -\frac{\partial P}{\partial X}-\frac{P r_{b f}\left(\mu_{n f} / \mu_{f}\right)}{D a\left[\phi\left(\rho_{p} / \rho_{f}\right)+(1-\phi)\right]} U \\
U \frac{\partial V}{\partial X}+V \frac{\partial V}{\partial Y}=- & \frac{C_{F}}{\sqrt{D a} \varepsilon^{3 / 2}} V \sqrt{U^{2}+V^{2}}+\frac{\operatorname{Pr}_{f}\left(\mu_{n f} / \mu_{f}\right)}{\phi\left(\rho_{p} / \rho_{f}\right)+(1-\phi)}\left(\frac{\partial^{2} V}{\partial X^{2}}+\frac{\partial^{2} V}{\partial Y^{2}}\right)-\frac{\partial P}{\partial Y} \\
& -\frac{\operatorname{Pr}\left(\mu_{n f} / \mu_{f}\right)}{D a\left[\phi\left(\rho_{p} / \rho_{f}\right)+(1-\phi)\right]} V+\frac{\phi \rho_{p}\left(\beta_{p} / \beta f\right)+(1-\phi) \rho_{f}}{\rho_{n f}} \operatorname{RaPr} \theta, \\
& U \frac{\partial \theta}{\partial X}+V \frac{\partial \theta}{\partial Y}=\frac{k_{e f f}}{k_{f}} \frac{\left(\rho C_{p}\right)_{f}}{\left(\rho C_{p}\right)_{n f}}\left(\frac{\partial^{2} \theta}{\partial X^{2}}+\frac{\partial^{2} \theta}{\partial Y^{2}}\right),
\end{aligned}
$$

The governing equations of Navier Stokes equations (1)-(3), the energy equation (4) are transformed into dimensionless forms using the following dimensionless variables:

$$
\begin{gathered}
X=\frac{x}{L}, \quad Y=\frac{y}{L}, \quad U=\frac{u L}{\alpha_{f}}, \quad V=\frac{v L}{\alpha_{f}}, \quad \theta=\frac{T_{n f}-T_{c}}{T_{h}-T_{c}}, \quad S=\frac{s}{L}, \quad k_{e f f}=\varepsilon k_{n f}+(1-\varepsilon) k_{s} \\
\operatorname{Pr}=\frac{v_{f}}{\alpha_{f}}, \quad R a=\frac{g \beta_{f}\left(T_{h}-T_{c}\right) L^{3}}{v_{f} \alpha_{f}}, \quad D a=\frac{K}{L^{2}}, \quad P=\frac{p L^{2}}{\rho_{f} \alpha_{f}^{2}} .
\end{gathered}
$$

The dimensionless boundary conditions of Eqs. (1)-(4) are as follows:

$$
\begin{gathered}
U=V=0, \theta=1 \text { on }(1-H) / 2 \leq X \leq(1+H) / 2, Y=0, \\
U=V=0, \frac{\partial \theta}{\partial Y}=0 \text { on } 0 \leq X \leq(1-H) / 2 \text { and }(1+H) / 2 \leq X \leq 1, Y=0, \\
U=V=0, \theta=0 \text { on } 1+(0.5 A(1-\cos (2 N \pi X)) 1-A / 2), 0 \leq Y \leq 1, \\
U=V=0, \theta=0 \text { on }-(0.5 A(1-\cos (2 N \pi X)) 1-A / 2), 0 \leq Y \leq 1, \\
U=V=0, \frac{\partial \theta}{\partial Y}=0 \text { on } 0 \leq X \leq 1, Y=1 .
\end{gathered}
$$

The local Nusselt number evaluated at the bottom horizontal wall, which is defined by:

$$
N u=-\frac{k_{e f f}}{k_{f}}\left(\frac{\partial \theta_{n f}}{\partial Y}\right)_{Y=0} .
$$

Finally, the average Nusselt number evaluated at the bottom horizontal wall of the cavity which is given by: 


$$
\overline{N u}_{n f}=\int_{\frac{1-H}{2}}^{\frac{1+H}{2}} N u_{n f} \mathrm{~d} X
$$

In dimensionless form, local entropy generation can be expressed as:

$$
S_{\mathrm{GEN}}=\frac{k_{e f f}}{k_{f}}\left[\left(\frac{\partial \theta}{\partial X}\right)^{2}+\left(\frac{\partial \theta}{\partial Y}\right)^{2}\right]+\frac{\mu_{n f}}{\mu_{f}} \varepsilon N_{\mu}\left\{2\left[\left(\frac{\partial U}{\partial X}\right)^{2}+\left(\frac{\partial V}{\partial Y}\right)^{2}\right]+\left(\frac{\partial U}{\partial Y}+\frac{\partial V}{\partial X}\right)^{2}\right\}+\frac{\varepsilon\left(U^{2}+V^{2}\right)}{D a}
$$

where, $N_{\mu}=\frac{\mu_{f} T_{0}}{k_{f}}\left(\frac{\alpha_{f}}{L(\Delta T)}\right)^{2}$ is the irreversibility distribution ratio and $S_{\mathrm{GEN}}=S_{\mathrm{gen}} \frac{T_{0}^{2} L^{2}}{k_{f}(\Delta T)^{2}}$.

The terms of Eq. 19 can be separated to the following form:

$$
S_{\mathrm{GEN}}=S_{\theta}+S_{\Psi}
$$

where $S_{\theta}$ and $S_{\Psi}$ are the entropy generation due to heat transfer irreversibility (HTI) and fluid friction irreversibility (FFI), respectively.

$$
\begin{gathered}
S_{\theta}=\frac{k_{e f f}}{k_{f}}\left[\left(\frac{\partial \theta}{\partial X}\right)^{2}+\left(\frac{\partial \theta}{\partial Y}\right)^{2}\right] \\
S_{\Psi}=\frac{\mu_{n f}}{\mu_{f}} \varepsilon N_{\mu}\left\{2\left[\left(\frac{\partial U}{\partial X}\right)^{2}+\left(\frac{\partial V}{\partial Y}\right)^{2}\right]+\left(\frac{\partial U}{\partial Y}+\frac{\partial V}{\partial X}\right)^{2}\right\}+\frac{\varepsilon\left(U^{2}+V^{2}\right)}{D a}
\end{gathered}
$$

By Integrating Eq. 16 over the domain, the global entropy generation (GEG) for the present two-dimensional study is obtained

$$
\mathrm{GEG}=\int S_{\mathrm{GEN}} \mathrm{d} X \mathrm{~d} Y=\int S_{\theta} \mathrm{d} X \mathrm{~d} Y+\int S_{\Psi} \mathrm{d} X \mathrm{~d} Y
$$

It is appropriate to mention Bejan number in order to determine which one is the dominant, heat transfer or fluid friction irreversibility. Bejan number is defined as:

$$
B e=\frac{\int S_{\theta} \mathrm{d} X \mathrm{~d} Y}{\int S_{\mathrm{GEN}} \mathrm{d} X \mathrm{~d} Y}
$$

When $B e>0.5$, the HTI is the dominant, while when $B e<0.5$, the FFI is the dominant.

The governing dimensionless equations (1)-(4) subject to the boundary conditions (6)-(10) are solved with Galerkin weighted residual finite element method [11]. The computational domain is discretised into triangular elements. Triangular Lagrange finite elements of different orders are used for each of the flow variables within the computational domain. Residuals for each conservation equation are obtained by substituting the approximations into the governing equations. To simplify the nonlinear terms in the momentum equations, a Newton-Raphson iteration algorithm was used [11]. 


\section{Results and Discussion}

In this section, we present numerical results for the streamlines, isotherms and isentropic with various values of Darcy number $\left(10^{-6} \geq D a \geq 10^{-2}\right)$, nanoparticle volume fraction $(0 \geq \phi \geq 0.05)$ and amplitude of the wave $(0.05 \geq A \geq$ 0.3 ), where the values of Rayleigh number, porosity of the media, number of oscillations and Prandtl number are fixed at $R a=10^{6}, \varepsilon=0.5, N=3$ and $\operatorname{Pr}=6.2$. The values of the average Nusselt number are calculated for various values of $D a$ and $\phi$. The thermophysical properties of the base fluid (water) and solid Cu phases are tabulated in Table 1 .

Table 1: Thermo-physical properties of water and $\mathrm{Cu}$ nanoparticles.

\begin{tabular}{|c|c|c|}
\hline Physical properties & Fluid phase (water) & $\mathrm{Cu}$ \\
\hline$C_{p}$ & 4179 & 383 \\
\hline$\rho$ & 997.1 & 8954 \\
\hline$k$ & 0.6 & 429 \\
\hline$\mu$ & $21 \times 10^{-5}$ & $5.4 \times 10^{-5}$ \\
\hline
\end{tabular}

Figure 2 illustrates the effects of various Darcy number $(D a)$ on the streamlines, isotherms and isentropic maps for $\phi=0.03$ and $A=0.15$. At low Darcy number $\left(D a=10^{-5}\right)$, the flow within the cavity is characterized by two streamlines cells at the lower part of the cavity affected by the low velocities that prevail in the cavity. The intensity of the isotherm patterns decreases next to the cold wavy walls while in the middle of the cavity the patterns tend to take carved lines. The entropy generation in the cavity is primarily due to heat transfer irreversibility and it is almost negligible near the upper portion of the cavity due to insignificant heat flow in that region because of the slow velocity. With the increasing of $D a$ the permeability of the porous medium is increased and thus the fluid flow is intense resulting in enhanced convective heat from the hot bottom wall. At this stage, due to stronger fluid flow, the entropy generation is clearly higher compared to that for low Darcy number and the upper of the cavity participates in the entropy generation. This is because as the fluid circulation is stronger for higher $D a$ which leads to a high velocity gradients and therefore a more entropy generation due to fluid friction. However, the density of the entropy generation tends to decrease within the centre of the cavity due to the fact that most of the energy moves toward the wavy vertical walls. 

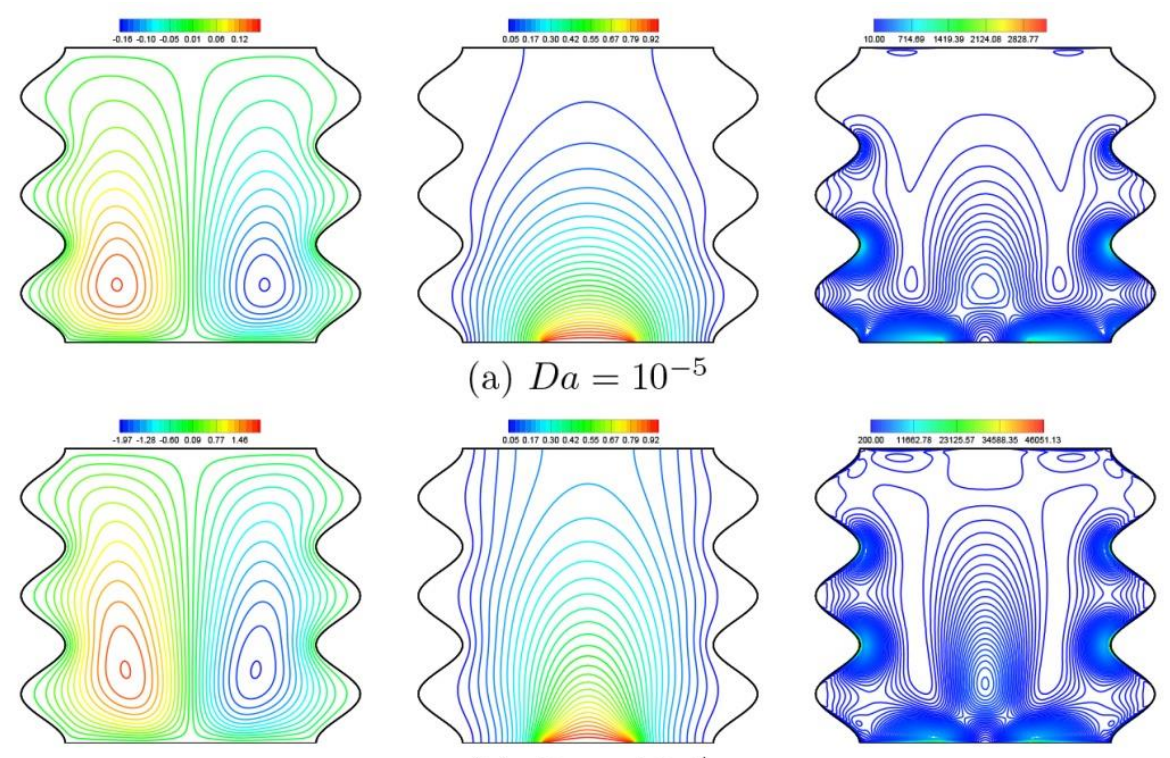

(a) $D a=10^{-5}$
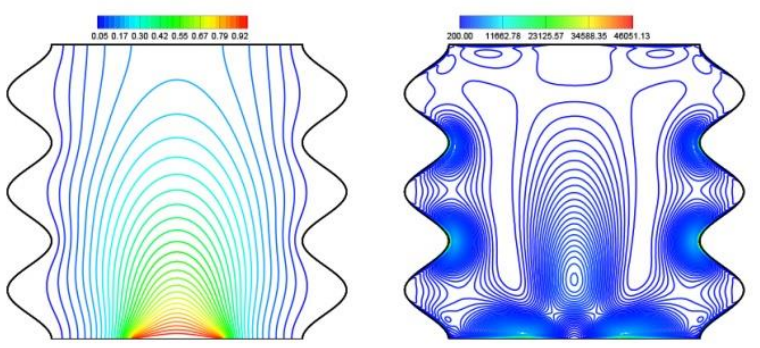

(b) $D a=10^{-4}$
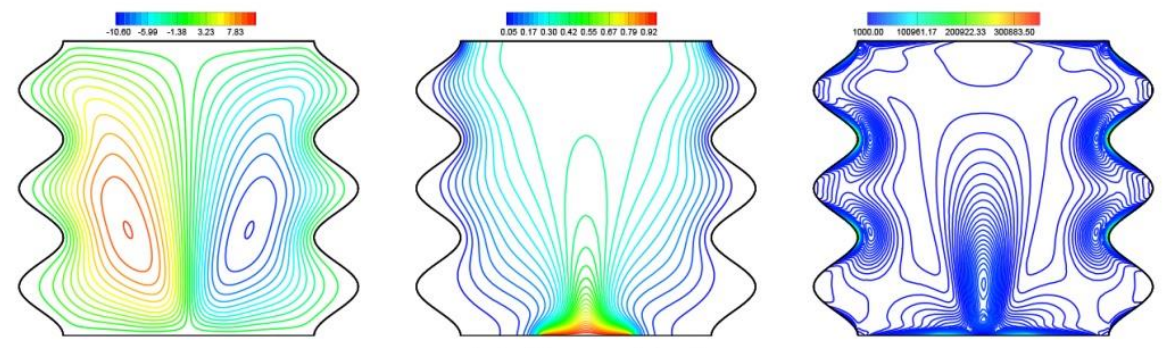

(c) $D a=10^{-3}$
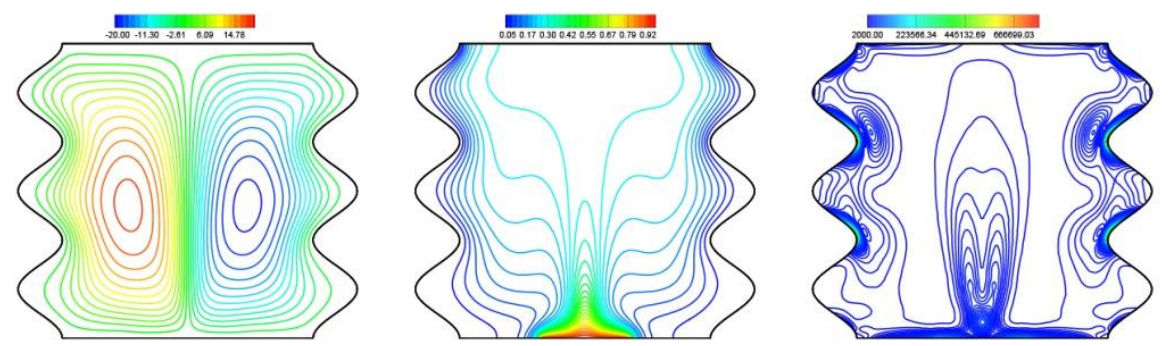

(d) $D a=10^{-2}$

Fig. 2: Variation of the streamlines (left), isotherms (middle), and isentropic (right) evolution by Darcy number ( $D a$ ) for $\phi=0.03$ and $A=0.15$.

Figure 3 illustrates the effects of various amplitude of the wave $(A)$ on the streamlines, isotherms and isentropic maps for $D a=10^{-3}$ and $\phi=0.03$. It is clearly observed from this figure that the amplitude of the wave has an influence on the geometric shape of the flow cell, as well as on the distribution of isotherms and isentropic lines. The flow within the cavity appears with two streamlines cells at the lower part of the cavity. The isotherm patterns observe with high intensity next the wavy walls of the cavity. The isentropic distribution shows a weak behaviour within the cavity, isentropic lines appear with high density within the wavy cavity affected by the lower thermal gradient. The intensity of the streamlines increases with the increasing of the amplitude of the wave, and as a result, the strength of the flow circulation increases. The intensity of the isotherm patterns decreases next the wavy wall with the increment of the amplitude due to the reduction of the gradient of the boundary layer. Increasing the amplitude of the wave up to the high values is clearly enhanced the entropy generation due to the strong fluid circulation. 

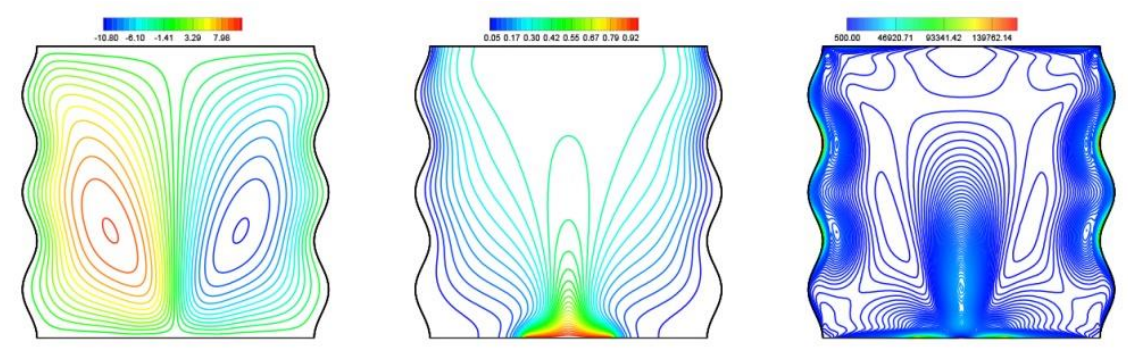

(a) $A=0.05$
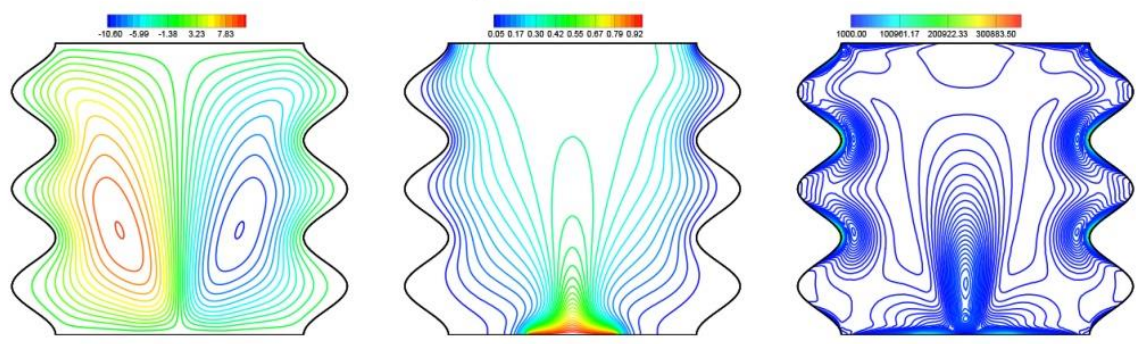

(b) $A=0.15$
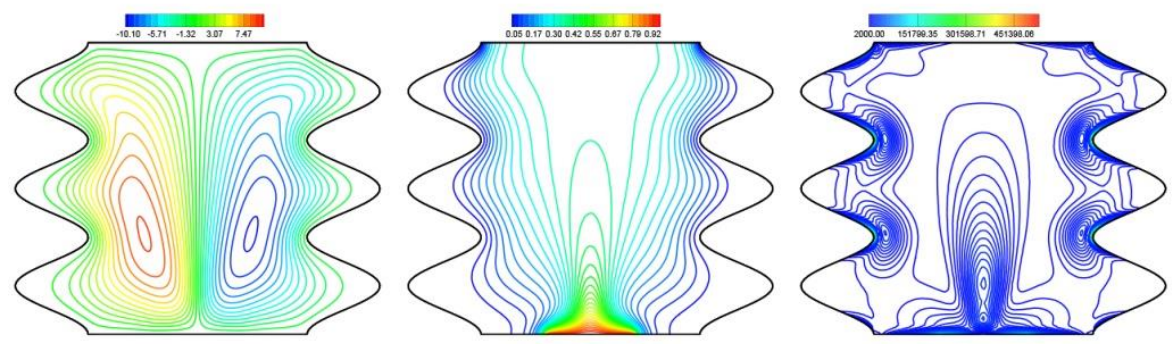

(c) $A=0.25$

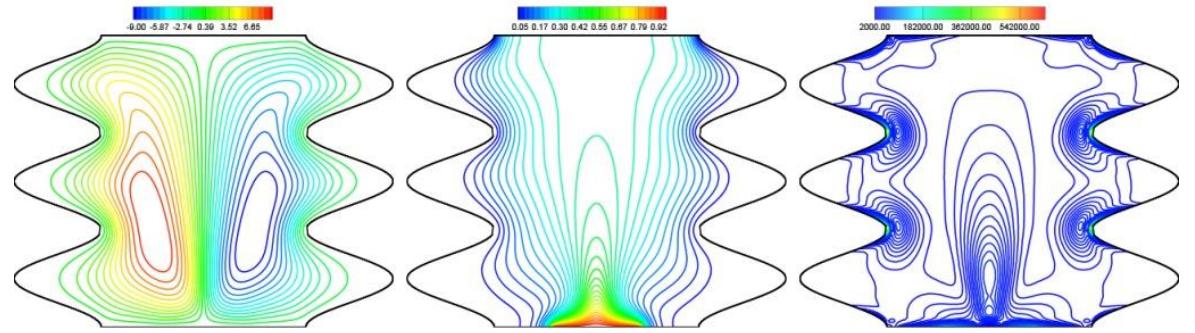

(d) $A=0.3$

Fig. 3: Variation of the streamlines (left), isotherms (middle), and isentropic (right) evolution by amplitude of the wave $(A)$ for $D a=10^{-3}$ and $\phi=0.03$.

Figure 4(a) shows the effects of various Darcy numbers on the local Nusselt number and along the heated part of the bottom wall for $\phi=0.03$ and $A=0.15$. Increasing Darcy tends to increase the gradient of the boundary layer and as a result, the local heat transfer enhances. Higher heat transfer enhancement is obtained with higher Darcy number. Figure 4(b) presents the effects of various nanoparticle volume fraction on the local Nusselt number and along the heated part of the bottom wall for $D a=10^{-3}$ and $A=0.15$. The local heat transfer increases by the augmentation of nanoparticle volume fraction due to the higher thermal conductivity of the nanoparticles. 


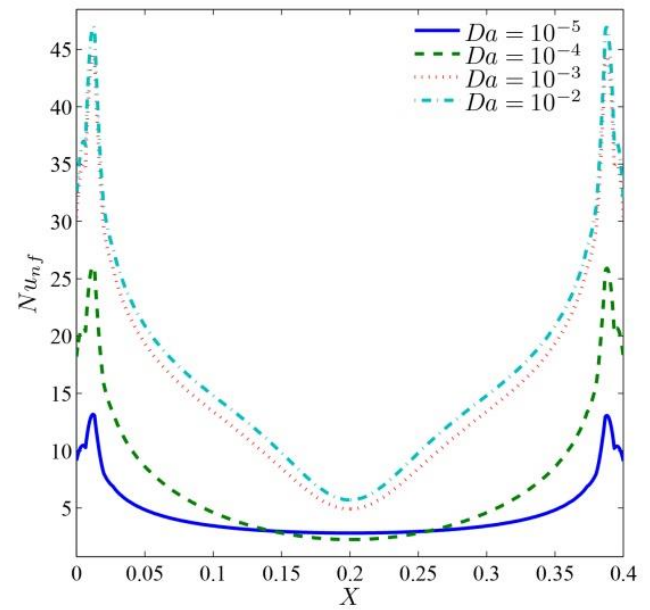

(a)

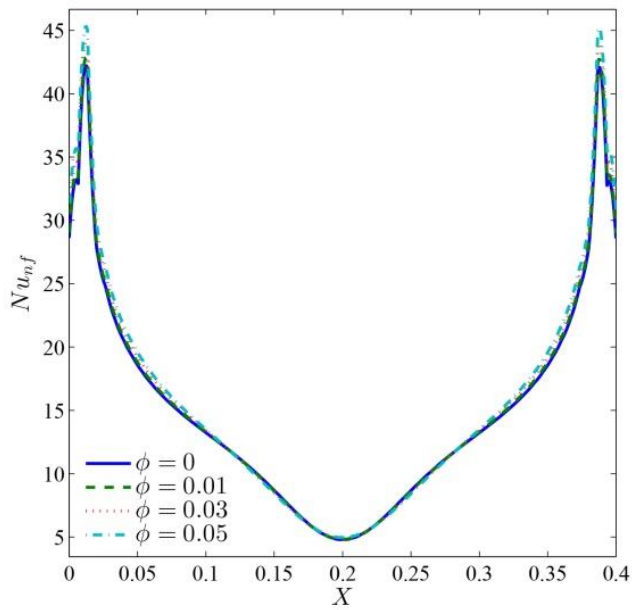

(b)

Fig. 4: Variations of local Nusselt numbers interface with $X$ for different (a) $D a$ and (b) $\phi$ at $A=0.15$.

Figure 5(a) shows the effects of nanoparticle volume fraction on the average Nusselt number with Darcy number for $A=0.15$. The heat transfer rate is clearly enhanced with increasing nanoparticle volume fraction. The values of average Nusselt number denote that the heat transfer rate increases and then decreases and increases after a while with the increment of nanoparticle volume fraction. Due to the various flow velocity speeds with different amount of nanoparticles. Figure 5(b) illustrates the effects of various values of the nanoparticle volume fraction on Bejan number with Darcy number. The average Bejan number tends to decrease as the Darcy number increases which clarify the dominance of the irreversibility due to the fluid friction with the high Darcy numbers.

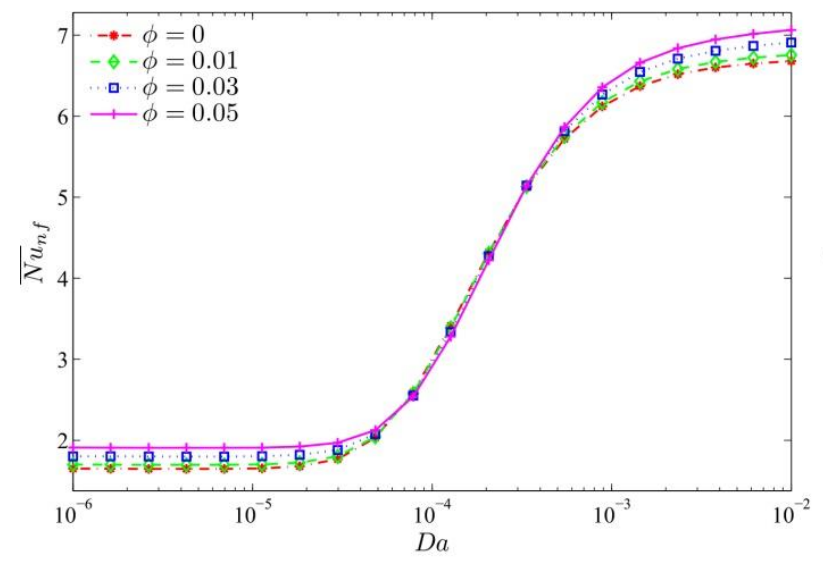

(a)

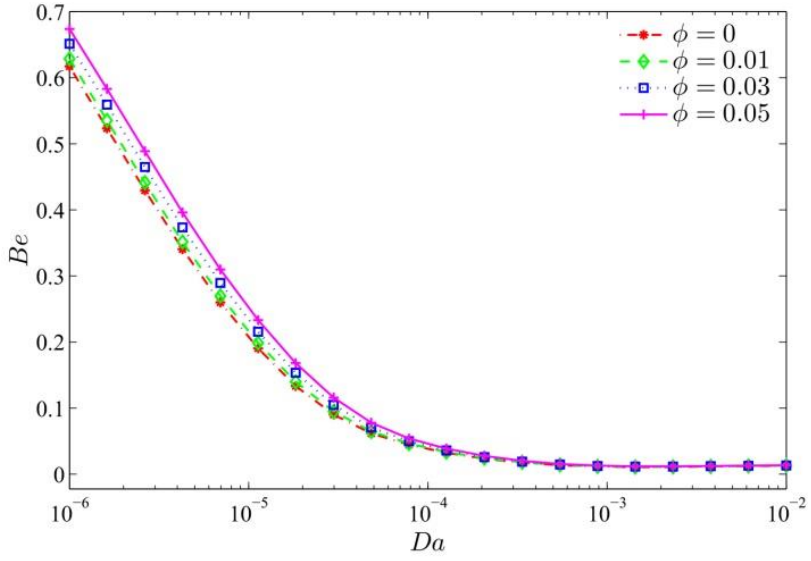

(b)

Fig. 5: Variation of (a) average Nusselt number and (b) Bejan number (Be) with Da for different $\phi$ at $A=0.15$.

\section{Conclusion}

The present study considered the natural convection and entropy generation in a porous wavy cavity saturated by a nanofluid with partial bottom temperature. The governing equations with the boundary conditions are solved numerically using the finite element method. The Forchheimer-Brinkman-extended Darcy and Tiwari and Das' nanofluid models are assumed to hold. Based on the obtained results, it is found that the strength of the streamlines increases with the increment of the Darcy number. The isotherms appear with high density close to the wavy walls. The entropy generation looks very strong with high values of the Darcy number. However, the density of the entropy generation tends to decrease within the centre of the cavity due to the fact that most of the energy moves toward the wavy vertical walls. The heat transfer rate is clearly enhanced with the increasing of the nanoparticle volume fraction and the Darcy number due to the increasing of the 
thermal conductivity. On the other hand, Bejan number reduces by the increment of the Darcy number when the fluid friction irreversibility (FFI) is the dominant.

\section{Acknowledgements}

The first and third authors thank Prince Mohammad Bin Fahd University for the financial support of this work under an internal grant project.

\section{References}

[1] A. Narasimhan, Essentials of heat and fluid flow in porous media. CRC Press, Boca Raton, 2013.

[2] A. Shenoy, M. Sheremet, I. Pop, Convective flow and heat transfer from wavy surfaces: Viscous fluids, porous media, and nanofluids. CRC Press, Taylor \& Francis Group, Boca Raton,, 2016.

[3] A. I. Alsabery, A. J. Chamkha, I. Hashim and P. G. Siddheshwar, "Effects of nonuniform heating and wall conduction on natural convection in a square porous cavity using LTNE model," Journal of Heat Transfer, vol. 139, no. 12 , p. $122008,2017$.

[4] P. K. Das and S. Mahmud, "Numerical investigation of natural convection inside a wavy enclosure," International Journal of Thermal Sciences, vol. 42, pp. 397-406, 2003.

[5] J. Rostami, "Unsteady natural convection in an enclosure with vertical wavy walls," Heat and Mass Transfer, vol. 44, pp. 1079-1087, 2008.

[6] M. Mansour, M. A. El-Aziz, R. Mohamed and S. E. Ahmed, "Numerical simulation of natural convection in wavy porous cavities under the influence of thermal radiation using a thermal non-equilibrium model," Transport in Porous Media, vol. 86, 585-600, 2011.

[7] S. Qiang, I. Pop, "Free convection in a triangle cavity filled with a porous medium saturated with nanofluids with flush mounted heater on the wall," International Journal of Thermal Sciences, vol. 50, no. 11, pp. 2141-2153, 2011.

[8] A. J. Chamkha and M. A. Ismael, "Conjugate heat transfer in a porous cavity filled with nanofluids and heated by a triangular thick wall," International Journal of Thermal Sciences, vol. 67, pp. 135-51, 2013.

[9] E. Abu-Nada, A. J. Chamkha, "Mixed convection flow of a nanofluid in a lid-driven cavity with a wavy wall," International Communications in Heat and Mass Transfer, vol. 57, pp. 36-47, 2014.

[10] M. Sheremet, D. Cimpean and I. Pop, "Free convection in a partially heated wavy porous cavity filled with a nanofluid under the effects of brownian diffusion and thermophoresis," Applied Thermal Engineering, vol. 113, pp. 413-418, 2017.

[11] A. I. Alsabery, M. A. Ismael, A. J. Chamkha and I. Hashim, "Mixed convection of $\mathrm{Al}_{2} \mathrm{O}_{3}$-water nanofluid in a double lid-driven square cavity with a solid inner insert using Buongiorno's two-phase model," International Journal of Heat and Mass Transfer, vol. 119, pp. 939-961, 2018. 